\title{
QTe Interval Prolongation in Drug Resistant-Tuberculosis Patients Treated with Shorter Treatment Regimen
}

\author{
Tutik Kusmiati $^{1}$, Yulia Devina Suci², Kristin Purnama Dewi², Soedarsono ${ }^{3}$ \\ ${ }^{1}$ Medical Staff, Infection Division, Departement of Pulmonology and Respiratory Medicine, Faculty of Medicine, \\ Universitas Airlangga-Dr. Soetomo General Academic Hospital, Surabaya, Indonesia, ${ }^{2}$ Resident, Departement \\ of Pulmonology and Respiratory Medicine, Faculty of Medicine, Universitas Airlangga-Dr. Soetomo General \\ Academic Hospital, Surabaya, Indonesia, ${ }^{3}$ Assistance Professor, Infection Division, Departement of Pulmonology \\ and Respiratory Medicine, Faculty of Medicine, Universitas Airlangga-Dr. Soetomo General Academic Hospital,
}

Surabaya, Indonesia

\begin{abstract}
Background: Shorter Treatment Regimen (STR) is a combination of treatments with a shortened period from 20-24 months to 9-11 months. Shortened treatment requires a higher dose of drug to kill resistant bacteria. Corrected QT (QTc) interval prolongation is one of the severe side effects of treatment.

Objective: This study aimed to find the factors of QTc prolongation in Drug Resistant-Tuberculosis (DRTB) patients treated with STR during an intensive phase.

Method: An analytical retrospective study was conducted at Dr. Soetomo General Academic Hospital, Surabaya, Indonesia. DR-TB patients on the STR regimen were collected based on medical records between September 2017 to August 2018. QTc interval was calculated by Fredericia formula. The relationship between QTc interval at baseline and occurrence of QTc prolongation was analyzed using Chi-Square of fisher's exact test.

Results: Among 108 patients on the STR regimen, there were 20 (28\%) patients experienced moderate QTc prolongation (471-500 ms), and 31 (28\%) patients had severe QTc prolongation ( $>500 \mathrm{~ms}$ ) during four months observation period in STR treatment. The prolonged QTc interval was significantly related to QTc interval at baseline $(\mathrm{p}=0.001)$. The QTc interval at baseline correlated significantly with the start time of QTc prolongation $(\mathrm{p}<0.001)$. Risk factors of age, gender, comorbid, hypertension, and potassium level at baseline had a significant negative correlation to QTc prolongation.
\end{abstract}

Conclusion: The prolonged QTc interval was significantly related to QTc interval at baseline. The QTc interval at baseline correlates significantly with the start time of QTc prolongation.

Keyword: Prolonged QTc, Drug Resistant-Tuberculosis, Shorter Treatment Regimen.

\section{Corresponding Author:}

\section{Tutik Kusmiati}

Departement of Pulmonology and Respiratory

Medicine, Faculty of Medicine, Universitas Airlangga -

Dr. Soetomo General Academic Hospital, Jalan Mayjen

Prof. Dr. Moetopo 6 - 8, Airlangga, Gubeng, Surabaya

60286, Indonesia

e-mail: tut.kusmiati@gmail.com

Phone: $+6231-5501656 /+6281803004561$

\section{Introduction}

Drug Resistant-Tuberculosis (DR-TB) is known for long-duration treatment (20-24 months) and unsatisfactory outcomes. Five hundred fifty-eight thousand new cases of DR-TB emerge each year globally, with success rate only $55 \%^{(1)}$. In May 2016, World Health Organization (WHO) recommended Shorter DR-TB Regimen (9-11 months) with a success rate of 84\% (95CLs:79\%-87\%) $)^{(2)}$. Indonesia start adopting and implementing a shorter DR-TB treatment regimen 
(STR) in September 2017. The Indonesia National TB Program is expecting STR can increase enrollment and success rate of treatment, also giving better outcomes rather than long-duration regimen ${ }^{(3)}$.

The STR contains new drugs such as a high dose of Moxifloxacin ${ }^{(3)}$. Moxifloxacin becomes an essential drug in the treatment of resistant tuberculosis ${ }^{(4)}$ that causes high bactericidal activity ${ }^{(5)}$. Nevertheless, these drugs have the ability to delay cardiac repolarization, represent as corrected QT (QTc) interval prolongation on electrocardiogram (ECG) ${ }^{(6)}$. QTc interval prolongation can disrupt normal cardiac rhythms and lead to fatal arrhythmias such as torsades de pointes ${ }^{(7)}$ and lead to sudden cardiac death ${ }^{(8)}$. Thus, TB program recommends monitoring QTc interval to ensure patient safety and optimum patient outcome ${ }^{(3)}$.

The risk of drug-induced QT interval prolongation appears to be frequently overlooked in clinical practice $^{(8)}$. A literature review of 249 patients with TdP associated with non-cardiac QTc-prolonging drugs reported that, apart from the drugs, $71 \%$ had at least two other risk factors $^{(9)}$. Clinically significant QTcF changes (QTcF $>500 \mathrm{~ms}$ or an increase $60 \mathrm{~ms}$ ) were observed in $10 / 60$ patients $(17 \%, 95 \% \mathrm{CI} ; 8.0-30.7)$ without clinical events $^{(10)}$.

However, there is not enough information regarding the relationship between QTc interval baseline, the occurrence of QTc prolongation among DR-TB patients on STR regimen, the dosage of moxifloxacin with QTc prolongation, and outcome treatment. This study aimed to find the factors of QTc prolongation in DR-TB patients treated with STR during the intensive phase.

\section{Method}

A descriptive retrospective study was conducted in 123 DR-TB patients treated with STR from September 2017 to August 2018 at Dr. Soetomo General Academic Hospital, Surabaya, Indonesia. Only 108 patients were eligible for this study. DR-TB was confirmed by rapid molecular test XpertMTB/RIF and Drugs Sensitivity Test (DST). This study reviewed the medical records of patients with STR during the intensive phase.

STR is a 9-month regimen consisting of kanamycin, ethionamide, moxifloxacin, clofazimine, ethambutol, and high dose isoniazid ${ }^{(3)}$. The intensive phase lasts a minimum of four months and could be extended up to 6 months because of delayed sputum smear convertion ${ }^{(11)}$.
This study obtained data on demographics, comorbidities, potassium $(\mathrm{K}+)$ baseline, baseline QTc interval, serial QTc interval, and the onset of QTc prolongation. Patient with missing serial ECG, incomplete medical record, and less than six-month treatment were excluded from this study.

Patient routinely has a 12-lead ECG before starting the treatment and each month when begin taking medication. QT interval defines as the time from the beginning of ventricular depolarization to completion of repolarization ${ }^{(7)}$. Measurement of the QT should be based on leads that normally show the earliest QRS onset and the latest end of the $\mathrm{T}$ wave (T-wave offset), which are II and V5. The end of the QT interval is the point at which the $\mathrm{T}$ wave reaches the iso-electric line ${ }^{(12)}$.

Baseline QTc was evaluated though first ECG recording before taking STR regimen therapy. QTc interval was measured from the onset of $\mathrm{Q}$ wave of QRS complex to the end of the T wave ${ }^{(13)}$. Frederica formula $\left(\mathrm{QTcFri}=\mathrm{QT} / \mathrm{RR}^{1 / 3}\right)$ was employed to count QT corection ${ }^{(14)}$.

QTc $>500 \mathrm{~ms}$ is considered as severe prolongation; QTc 471-500 ms is considered as moderate prolongation that requires further evaluation ${ }^{(15)}$. QTc prolongation was classified according to the Common terminology criteria for adverse events (CTCAE) guidelines version 4.03 (grade 0, QTc < 450; grade 1, QTc 450-479 ms; grade 2, QTc 480-499 ms; grade 3, QTc $>500 \mathrm{~ms}$; grade $4, \mathrm{QTc}>500 \mathrm{~ms}$ with life-threatening signs or symptoms; grade 5 , death) ${ }^{(16)}$.

Patient data were collected via Microsoft Excel and analyzed using IBM SPSS statistic 20.0 (IBM Corp., Armonk, NY, USA). A Multivariate logistic regression model was used to determine a risk factor relating to baseline QTc. The relationship between baseline QTc interval and occurrence of QTc prolongation was analyzed using Chi-Square of fisher's exact test. P-values $<0.05$ were considered statistically significant.

\section{Results}

Of 123 patients with STR, only 108 patient were eligible for this study that consisted of $63(58.3 \%)$ males and $45(41.7 \%)$ females. In this study, patients were divided into two groups, normal QTc and prolonged QTc groups. There were 57 (53\%) patients with normal QTc $(<470 \mathrm{msc})$ and $51(47 \%)$ patients with prolonged QTc ( $>470 \mathrm{msc})$. Thirty (28\%) patients had severe 
QTc prolongation (QTc > $500 \mathrm{~ms}$ ), and 21(19\%) had moderate QTc prolongation (QTc between 470-500 ms).

The demographic data from this study were noted in Table 1 . The mean age patient was $46.4 \pm 12.1$. There were $42(38.9 \%)$ patients with diabetes mellitus and 8 patients with hypertension (7.4\%). The mean of kalium at baseline was $4.08 \pm 0.6$. Based on multiple regression model, there was no significant correlation between age, gender, diabetes mellitus, potassium at baseline, and smoking $(\mathrm{p}>0.05)$.

Baseline QTc was divided into four groups (Table 2). There were 10 patients with baseline QTc with $>470 \mathrm{msc}, 15$ patients with QTc 451-470, 73 patients with QTc 400-450, and 10 patients with QTc $<400 \mathrm{~ms}$. The results of chi-square of fisher exact showed that baseline QTc had a significant correlation with QTc interval prolongation after taking STR $(p=0.001)$.

This study found that baseline QTc had a significant correlation with the onset of QTc $(\mathrm{p}<0.001)$. From 10 patients, $9(90 \%)$ patients had QTc prolongation at first month after taking STR drugs and one patient $(10 \%)$ in the second month. All patients developed into QTc prolongation as seen at Table 3. From baseline QTc 451-470, 2 (13.3\%) patients had QTc prolongation at first month, and $3(20 \%)$ patients had QTc prolongation at second month, and $6(40 \%)$ patients did not develop into QTc prolongation. Forty-six patients with baseline QTc 400 - $450 \mathrm{~ms}$ did not have prolonged QTc during the intensive phase $(63 \%)$, and $7(70 \%)$ patients from baseline QTc $<400 \mathrm{msc}$ did not have prolonged QTc.

Of 109 patients with moxifloxacin, six $(10.5 \%)$ patients with normal QTc had $400 \mathrm{mg}$ oral moxifloxacin, $29(50.9 \%)$ had $600 \mathrm{mg}$ oral moxifloxacin, and 22 (38.6\%) had $800 \mathrm{mg}$ oral moxifloxacin. There were fifteen (71.4\%) patients with moderate prolong QTc (471-500) had $600 \mathrm{mg}$ oral moxifloxacin, 18 (58.1\%) patients with severe prolong QTc $>500 \mathrm{~ms}$ had 600 $\mathrm{mg}$. Almost all patients took $600 \mathrm{mg}$ oral moxifloxacin (Table 4). There was no significant difference in the dosage of moxifloxacin with QTc interval prolongation.

There was a significant correlation between outcome therapy with QTc prolongation ( $\mathrm{p}<0.001$; table 5$)$. A favorable outcome was cured, while an adverse was defined as death. There were $22(47.8 \%)$ patients on treatment had severe QTc prolongation $(>500 \mathrm{~ms}), 8$ (44.4\%) death patients had severe QTc prolongation and they were more likely died because of sudden death.
Compared with moderate QTc (471-500msc), 6 (13\%) patients on treatment STR and $6(28.6 \%)$ died.

\section{Discussion}

This study found that $47 \%$ patients with STR treatment developed into QTc prolongation. Cellular mechanism of QTc prolongation involves inhibition of rapid component of the delayed rectifier potassium current (IKr). Locking IKr leads to prolongation of the ventricular action potential duration, leading to an excess sodium influx or a decreased potassium efflux (17). This excess of positively interaction with the ion channels is responsible for myocardial contractility that leads to an extended repolarization phase ${ }^{(18)}$, resulting in a prolonged QT interval and causing arrhythmias such as Torsades de Pointes ${ }^{(17)}$.

Table 1 showed that age, gender, diabetes mellitus, hypertension, smoking, and potassium at baseline had negative correlations with QTc prolongation. Therefore, the incidence of QTc prolongation might have been caused by the use of QT drugs. In this study, moxifloxacin was the strongest predictor of druginduced QTc prolongation. Moxifloxacin inhibition of $\mathrm{hERG} / \mathrm{IKr}$ occurred at concentrations higher than those observed clinically during treatment ${ }^{(19)}$.

A recent study demonstrated that the risk of cardiac arrest in hospitalized patients with several underlying diseases was increased two-times with the use of non-antiarrhythmic QT-prolonging drugs. The risk of cardiac arrest is higher if receiving more than one daily dose if treated with more than one QT-prolonging drug, and with drugs that interfere with the metabolism or elimination of the QT-prolonging agent ${ }^{(20)}$. The individual variability in drug sensitivity and the variable influence of factors that affect QTc prolongation on each patient's drug exposure (e.g., dose, drug metabolism, and route of administration) might reduce the predictive accuracy of study ${ }^{(21)}$.

Moxifloxacin $400 \mathrm{mg}$ is known to cause a mean increase in the QTc interval of between 10 and $14 \mathrm{~ms}$ in 2-4 hours after a single oral dose. In addition, a supratherapeutic dose of moxifloxacin $(800 \mathrm{mg})$ results in a nearly two-times increase in the QTc interval from baseline compared with the 400-mg dose ${ }^{(22)}$. Unfortunately, this study did not mention $600 \mathrm{mg}$ dose.

Baseline QTc shown in Table 2 indicated that patients with longer baseline QTc significantly would develop 
into prolonged QTc. Increased $10 \mathrm{~ms}$ from baseline on QTc interval results in 6\% increase for the risk of a cardiac event. The risk of $\mathrm{TdP}$ also increases when the QTc interval lengthens more than $60 \mathrm{~ms}$ compared with the baseline value ${ }^{(19)}$ Therefore, physicians should anticipate this possible increase in QTc intervals and perform ECGs before treatment to identify baseline QTe which may be the result of drug-induced Long QT Syndrome ${ }^{(16)}$.

Treated patient with QTc Baseline $>470 \mathrm{msc}$ more likely developed QTc prolongation at first month of administered drugs (Table.3). Drug-induced QTc prolongation can occur at different times while the patient is receiving offending oral agent, as it usually corresponds with the expected time of the medication's peak concentration (17). An increase in plasma moxifloxacin concentration is associated with QTc prolongation ${ }^{(23)}$.

The regulatory of QTc study is identified by the EMA, the US FDA, and the International Conference on Harmonisation (ICH E14) of Technical Requirements for Registration of Pharmaceuticals for Human Use as a positive control in thorough QTe studies ${ }^{(24)}$. The FDA further concludes that the risk of arrhythmias appears to increase with the extent of QT/QTc prolongation ${ }^{(6)}$.

Moxifloxacin, a new generation of fluoroquinolone, has been shown to have better activity against mycobacterium tuberculosis than floxacin ${ }^{(25)}$. Moxifloxacin is an 8-methoxy quinolone antimicrobial drug, which is often used as a positive control in thorough QT (TQT) studies (26). Moxifloxacin is a reversible blocker of the rapid component of the delayed rectifier, potassium current of the cardiac Ikr potassium channel and causes a mean increase of the QTc interval of 10-14 ms between 2 and 4 hours after a single oral dose of $400 \mathrm{mg}{ }^{(27)}$. Moxifloxacin binds to and inhibits the human ether-a-go-go-related gene (hERG) $\mathrm{IKr} \alpha$ subunit and thereby prolongs the cardiac repolarization interval. Patch-clamp studies indicate that moxifloxacin can bind with high affinity to the open $\mathrm{IKr}$ and block the conductance $^{(28)}$.

The direct inhibition of hERG channel is the bestcharacterized mechanism by which drugs can inhibit cardiac repolarization ${ }^{(28)}$. However, other inhibitory mechanisms, such as the IKr, interfere with hERGprotein intracellular trafficking or promoting the degradation of this protein and action on the autonomic nervous system or the sinoatrial node ${ }^{(19)}$. Drugs block the delayed rectifier potassium channel, which is coded by human ether-a-go-go-related gene (hERG). The distinct molecular structure of the hERG channel makes it more susceptible to medications. IKr currently plays an important role in phase-3 of ventricular action potential (ventricular repolarization) ${ }^{(29)}$.

The pharmacokinetics of this moxifloxacin make it suitable to be an anti-TB drug. The oral dosing achieves a peak serum concentration of $>4 \mathrm{mg} \cdot \mathrm{L}-1$. Maximum concentration in serum and the area under the concentration (AUC)-time curve from 0 to $24 \mathrm{~h}$ has been reported as $3.4 \mathrm{mg} \cdot \mathrm{L}-1$ and $30.2 \mathrm{mg} \cdot \mathrm{h} \cdot \mathrm{L}-1$. Respectively, high values were found on day 10. Peak concentrations of the drug are achieved rapidly, with all patients achieving this within two hours. The half-life is reported as $\sim 12 \mathrm{~h}^{(5)}$.

Different absorption characteristics per dose were assumed, so a different parameter was estimated by dose in the absorption model. The absorption rate of $400 \mathrm{mg}$ moxifloxacin was faster than that of 800 $\mathrm{mg}^{(30)}$. Clinical vigilance and constant monitoring are important for all potential toxicities associated with high-dose moxifloxacin are imperative, especially for cardiotoxicity, which needs periodic electrocardiographic assessment throughout the treatment for all patients, with possibly 24 hours. Holter monitoring for selected cases is needed to comprehensively detect potentially dangerous arrhythmia ${ }^{(31)}$.

Yew and chang (2018) reported that $1-10 \%$ of patients with shorter MDR-TB treatment regimen containing high-dose moxifloxacin had QTc prolongation ${ }^{(31)}$. From systematic review, it summarises 15 years of research demonstrate that moxifloxacin is well absorbed orally and highly active against M. tuberculosis ${ }^{(5)}$.

QT prolongation has been described previously as a risk factor for all-cause mortality and more specifically, cardiovascular mortality ${ }^{(32)}$. On a study of 172 patients, the most common cause for QTc prolongation was QTc interval-prolonging medication and was deemed most responsible in $48 \%$ of patients, with $25 \%$ of these patients taking $\geq$ two offending drugs ${ }^{(29)}$. A study conducted by Haugaa et al. showed the death diagnosis itself does not directly reflect arrhythmic death. It is important to analyze clinical courses in the group of patients with a QTc interval of $500 \mathrm{~ms}$ or higher to fill the gap between the death diagnosis and the real number of patients with 
arrhythmia-related death ${ }^{(33)}$. Thomas et al. found that acutely ill patients with prolonged QT intervals had nearly a three-time odds ratio for an adverse event in ICU (34). Patients with clinically significant QT prolongation should undergo continuous ECG monitoring ${ }^{(35)}$.

In our study, 22 patients who were still undergoing STR and moxifloxacin treatment had severe QTc prolongation. A cohort study in South Korea in 373 patients receiving anti-tuberculosis drugs found $16 \%$ incidence of ECG abnormalities, and $0.8 \%$ presented with cardiac adverse events, The study concluded that, despite QTc prolongation, clinically meaningful events appeared to be minimal ${ }^{(18)}$. Survival curves of those with/without prolonged QTc separated well within 50 days of hospital admission ${ }^{(7)}$.

Table 1. Demographics data

\begin{tabular}{|l|c|c|c|c|}
\hline Characteristics & Total n (\%) & Normal QTe n (\%) & Prolong QTc n (\%) & p \\
\hline Age* & $46.4 \pm 12.1$ & $45.5 \pm 13.8$ & $47.5 \pm 9.7$ & 0.162 \\
\hline Gender & & & & \\
Male & $63(58.3 \%)$ & $39(68.4 \%)$ & $24(47.1 \%)$ & 0.278 \\
Female & $45(41.7 \%)$ & $18(31.6 \%)$ & $27(52.9 \%)$ & 0.836 \\
\hline Diabetes Melitus & & & & \\
Yes & $42(38.9 \%)$ & $20(35.1 \%)$ & $29(43.1 \%)$ & 0.946 \\
No & $66(61.1 \%)$ & $37(64.9 \%)$ & $4(7.8 \%)$ & \\
\hline Hypertension & & & $47(92.2 \%)$ & \\
Yes & $8(7.4 \%)$ & $4(7 \%)$ & & \\
No & $100(92.6 \%)$ & $53(93 \%)$ & $16(31.4 \%)$ & \\
\hline Smoking & & & $35(68.6 \%)$ & \\
Yes & $41(38 \%)$ & $25(43.9 \%)$ & $4.03 \pm 0.6$ & \\
No & $67(62 \%)$ & $32(56.1 \%)$ & & \\
\hline Kalium Baseline* & $4.08 \pm 0.6$ & $4,12 \pm 0.6$ & & \\
\hline
\end{tabular}

$*$ Mean \pm SD

Table 2. Correlation between baseline QTe and QTe interval prolongation

\begin{tabular}{|l|c|c|c|c|}
\hline Baseline & Severe QTc $>\mathbf{5 0 0}$ & Moderate QTc 471-500 & Normal QTc $<\mathbf{4 7 0}$ & p \\
\hline QTc $>470(n=10)$ & $6(60.0)$ & $3(30.0)$ & $1(10.0)$ & $0.001 *$ \\
\hline Tc 451-470 $(n=15)$ & $4(26.7)$ & $7(46.7)$ & $4(26.7)$ & $45(61.6)$ \\
\hline QTc 400-450 $(n=73)$ & $18(24.7)$ & $10(13.7)$ & $7(70.0)$ & \\
\hline QTc $<400(n=10)$ & $3(30.0)$ & $0(0.0)$ & \\
\hline
\end{tabular}

$* \mathrm{p}<0.05$

Table 3. Correlation between baseline QTe with prolong QTe onset in DR-TB Patients

\begin{tabular}{|l|c|c|c|c|c|c|c|}
\hline Baseline & Month 1 & Month 2 & Month 3 & Month 4 & Month 5 & No prolong QT & p \\
\hline QTc $>470(n=10)$ & $9(90.0)$ & $1(10.0)$ & $0(0.0)$ & $0(0.0)$ & $0(0.0)$ & $0(0.0)$ & $0.000^{* *}$ \\
\hline QTc 451-470 $(\mathrm{n}=15)$ & $2(13.3)$ & $3(20.0)$ & $1(6.7)$ & $2(13.3 \%)$ & $1(6.7)$ & $6(40.0)$ & $46(63.0)$ \\
\hline $\begin{array}{l}\text { Baseline QTc 400-450 } \\
(\mathrm{n}=73)\end{array}$ & $6(8.2)$ & $13(17.8)$ & $5(6.8)$ & $2(2.7 \%)$ & $1(1.4)$ & $7(70.0)$ & \\
\hline Baseline QTc <400 $(\mathrm{n}=10)$ & $1(10.0)$ & $1(10.0)$ & $0(0.0)$ & $1(10.0)$ & $0(0.0)$ & \\
\hline
\end{tabular}

$* * \mathrm{p}<0.001$ 
Table 4. Correlation between QTe prolongation with moxifloxacin dose

\begin{tabular}{|c|c|c|c|c|}
\hline & \multicolumn{3}{|c|}{ Dosage moxifloxacin } & \multirow{2}{*}{$\mathrm{p}$} \\
\hline & $400 \mathrm{mg}$ & $600 \mathrm{mg}$ & $800 \mathrm{mg}$ & \\
\hline Normal QTc $<470$ & $6(10.5)$ & $29(50.9)$ & $22(38.6)$ & 0.565 \\
\hline Moderate prolong QTc 471-500 & $2(9.5)$ & $15(71.4)$ & $4(19.0)$ & \\
\hline Severe prolong QTc $>500$ & $3(9.7)$ & $18(58.1)$ & $10(32.3)$ & \\
\hline
\end{tabular}

Table 5. Correlation between outcome therapy with QTe Prolongation

\begin{tabular}{|l|c|c|c|c|}
\hline & Severe QTc $>\mathbf{5 0 0}$ & Moderate QTc 471-500 & Normal QTc <470 & p \\
\hline Cured $(\mathrm{n}=9)$ & $1(11.1)$ & $4(44.4)$ & $4(44.4)$ & $0.000^{* *}$ \\
\hline Death $(\mathrm{n}=18)$ & $8(44.4)$ & $6(28.6)$ & $4(12.9)$ & $17(29.8)$ \\
\hline Dropout $(\mathrm{n}=20)$ & $3(15.0)$ & $0(0.0)$ & $10(62.5)$ & \\
\hline Lost to Follow Up (n=16) & $1(6.2)$ & $5(31.2)$ & $18(39.1)$ & \\
\hline On Treatment $(\mathrm{n}=46)$ & $22(47.8)$ & $6(13.0)$ & & \\
\hline
\end{tabular}

$* * \mathrm{p}<0.001$

\section{Conclusions}

Short term regimen DR-TB has the potential to prolong QT interval. The prolonged QTe interval is significantly related to the QTc interval at baseline. The QTc interval at baseline correlates significantly with the start time of QTc prolongation. This study presents different approaches that balance the need for life-saving regimens and medications, raise awareness of cardiac events, propose a strategy for ECG monitoring in STR DR-TB.

Conflict of Interest: The authors declare that they have no conflict of interest. The authors have written the ICMJE Authorship form.

Funding: None

Data Availability: The data set used and/or analyzed during the current study are available from corresponding author on reasonable request.

Ethics Statement: All procedures performed in studies involving human participants were in accordance with the ethical standards of the Ethics Committee in Dr. Soetomo General Academic Hospital, Surabaya, Indonesia (1491/KEPK/IX/2019).

List of Abbreviations: DR-TB $=$ Drug ResistantTuberculosis, STR $=$ Shorter, MFX $=$ Moxifloxacin, $\mathrm{ECG}=$ Electrocardiography, $\mathrm{WHO}=$ World Health Organization, DST $=$ Drug Sensitivity Test.

\section{References}

1. Organization WH. Global tuberculosis report 2018. Geneva: World Health Organization; 2018. Licence: CC BY-NC-SA 3.0 IGO. WHO/CDS/ TB/2018.20. Available from: http://apps. who. int/ iris/bitstream ...; 2018.

2. Organization WH. Frequently asked questions about the implementation of the new WHO recommendation on the use of the shorter MDR-TB regimen under programmatic conditions. Geneva, Switzerland: World Health Organization, 2016. 2016.

3. Waworuntu W, Subuh HM, Andriansyah, Karuniawati A, Pramadyani AH, Sonata B, et al. Petunjuk Teknis Pengobatan TB Resisten Obat dengan Paduan Jangka Pendek di Fasyankes TB Resistan Obat. Jakarta: Kementerian Kesehatan Republik Indonesia; 2017.

4. Kang BH, Jo K-W, Shim TS. Current Status of Fluoroquinolone Use for Treatment of Tuberculosis in a Tertiary Care Hospital in Korea. Tuberc Respir Dis (Seoul). 2017;80(2):143-52.

5. GillespieSH. Therole ofmoxifloxacinintuberculosis therapy. Eur Respir Rev. 2016;25(139):19-28.

6. Food, Administration D, Health UDo, Services H. Guidance for Industry. E14 Clinical Evaluation of QT/QTe Interval Prolongation and Proarrhythmic Potential for Non-Antiarrhythmic Drugs. http:// 
www fda gov/cder/guidance/6922fnl pdf. 2005.

7. Mahmud R, Gray A, Nabeebaccus A, Whyte MB. Incidence and outcomes of long QTc in acute medical admissions. Int $\mathrm{J}$ Clin Pract. 2018;72(11):e13250-e.

8. Buss VH, Lee K, Naunton M, Peterson GM, Kosari S. Identification of Patients At-Risk of QT Interval Prolongation during Medication Reviews: A Missed Opportunity? J Clin Med. 2018;7(12):533.

9. Harausz E, Cox H, Rich M, Mitnick CD, Zimetbaum P, Furin J. QTc prolongation and treatment of multidrug-resistant tuberculosis. Int J Tuberc Lung Dis. 2015;19(4):385-91.

10. Yoon HY, Jo KW, Nam GB, Shim TS. Clinical significance of QT-prolonging drug use in patients with MDR-TB or NTM disease. Int J Tuberc Lung Dis. 2017;21(9):996-1001.

11. Piubello A, Harouna SH, Souleymane MB, Boukary I, Morou S, Daouda M, et al. High cure rate with standardised short-course multidrug-resistant tuberculosis treatment in Niger: no relapses. Int $\mathbf{J}$ Tuberc Lung Dis. 2014;18(10):1188-94.

12. Porta-Sánchez A, Gilbert C, Spears D, Amir E, Chan J, Nanthakumar K, et al. Incidence, Diagnosis, and Management of QT Prolongation Induced by Cancer Therapies: A Systematic Review. J Am Heart Assoc. 2017;6(12):e007724.

13. Rabkin SW. Impact of Age and Sex on QT Prolongation in Patients Receiving Psychotropics. Can J Psychiatry. 2015;60(5):206-14.

14. VandenberkB, VandaelE, Robyns T, Vandenberghe J, Garweg C, Foulon V, et al. Which QT Correction Formulae to Use for QT Monitoring? J Am Heart Assoc. 2016;5(6):e003264.

15. Shenasa M, Josephson ME, Estes III NM. The ECG handbook of contemporary challenges: Cardiotext Publishing; 2015.

16. Kloth JSL, Pagani A, Verboom MC, Malovini A, Napolitano C, Kruit WHJ, et al. Incidence and relevance of QTc-interval prolongation caused by tyrosine kinase inhibitors. Br J Cancer. 2015;112(6):1011-6.

17. Li M, Ramos LG. Drug-Induced QT Prolongation And Torsades de Pointes. P T. 2017;42(7):473-7.

18. Monedero-Recuero I, Hernando-Marrupe L, Sánchez-Montalvá A, Cox V, Tommasi M, Furin J, et al. QTc and anti-tuberculosis drugs: a perfect storm or a tempest in a teacup? Review of evidence and a risk assessment. Int $\mathrm{J}$ Tuberc Lung Dis. 2018:10.5588/ijtld.18.0423.

19. Cubeddu LX. Drug-induced Inhibition and Trafficking Disruption of ion Channels: Pathogenesis of QT Abnormalities and Druginduced Fatal Arrhythmias. Curr Cardiol Rev. 2016;12(2):141-54.

20. Tomaselli Muensterman E, Tisdale JE. Predictive Analytics for Identification of Patients at Risk for QT Interval Prolongation: A Systematic Review. Pharmacotherapy. 2018;38(8):813-21.

21. Schwartz PJ, Woosley RL. Predicting the Unpredictable: Drug-Induced QT Prolongation and Torsades de Pointes. J Am Coll Cardiol. 2016;67(13):1639-50.

22. Moon SJ, Lee J, An H, Yim D-S, Chung J-Y, Yu $\mathrm{K}-\mathrm{S}$, et al. The effects of moxifloxacin on QTc interval in healthy Korean male subjects. Drugs $\mathrm{R}$ D. 2014;14(2):63-71.

23. Morganroth J, Wang Y, Thorn M, Kumagai Y, Harris S, Stockbridge N, et al. Moxifloxacininduced QTc interval prolongations in healthy male Japanese and Caucasian volunteers: a direct comparison in a thorough QT study. Br J Clin Pharmacol. 2015;80(3):446-59.

24. Haverkamp W, Kruesmann F, Fritsch A, van Veenhuyzen D, Arvis P. Update on the cardiac safety of moxifloxacin. Curr Drug Saf. 2012;7(2):149-63.

25. Chien J-Y, Chien S-T, Chiu W-Y, Yu C-J, Hsueh P-R. Moxifloxacin Improves Treatment Outcomes in Patients with Ofloxacin-Resistant MultidrugResistant Tuberculosis. Antimicrob Agents Chemother. 2016;60(8):4708-16.

26. Xu F-Y, Huang J-H, He Y-C, Liang L-Y, Li L-J, Yang J, et al. Population pharmacokinetics of moxifloxacin and its concentration-QT interval relationship modeling in Chinese healthy volunteers. Acta Pharmacol Sin. 2017;38(11):1580-8.

27. Taubel J, Ferber G, Lorch U, Batchvarov V, Savelieva I, Camm AJ. Thorough QT study of the effect of oral moxifloxacin on QTc interval in the fed and fasted state in healthy Japanese and Caucasian subjects. Br J Clin Pharmacol. 2014;77(1):170-9.

28. Täubel J, Ferber G, Fernandes S, Camm AJ. Diurnal Profile of the QTc Interval Following Moxifloxacin Administration. J Clin Pharmacol. 2019;59(1):3544. 
29. Arunachalam K, Lakshmanan S, Maan A, Kumar N, Dominic P. Impact of Drug Induced Long QT Syndrome: A Systematic Review. J Clin Med Res. 2018;10(5):384-90.

30. Hong T, Han S, Lee J, Jeon S, Park G-J, Park $\mathrm{W}-\mathrm{S}$, et al. Pharmacokinetic-pharmacodynamic analysis to evaluate the effect of moxifloxacin on QT interval prolongation in healthy Korean male subjects. Drug Des Devel Ther. 2015;9:1233-45.

31. Yew WW, Chang KC. Management of adverse reactions to high-dose moxifloxacin used in multidrug-resistant tuberculosis treatment programmes. Respirology. 2019;24(3):201-3.

32. Anderson HN, Bos JM, Haugaa KH, Morlan BW, Tarrell RF, Caraballo PJ, et al. Prevalence and Outcome of High-Risk QT Prolongation
Recorded in the Emergency Department from an Institution-Wide QT Alert System. J Emerg Med. 2018;54(1):8-15.

33. Mizusawa Y, Wilde AAM. QT Prolongation and Mortality in Hospital Settings: Identifying Patients at High Risk. Mayo Clinic Proceedings. 2013;88(4):309-11.

34. George TK, Chase D, Peter JV, Satyendra S, Kavitha R, George LR, et al. Association between a prolonged corrected QT interval and outcomes in patients in a medical Intensive Care Unit. Indian $\mathrm{J}$ Crit Care Med. 2015;19(6):326-32.

35. Thomas SHL, Behr ER. Pharmacological treatment of acquired QT prolongation and torsades de pointes. Br J Clin Pharmacol. 2016;81(3):420-7. 